\title{
The accuracy of spatial information from temporally and spatially organized mental maps
}

\author{
JACQUELINE M. CURIEL and GABRIEL A. RADVANSKY \\ University of Notre Dame, Notre Dame, Indiana
}

\begin{abstract}
The way a space is learned can result in a mental map that is either temporally or spatially organized (Curiel \& Radvansky, 1998). The present study examined the availability of spatial information under map learning conditions where either temporal or spatial organization has been previously observed. The finding was that people were fairly accurate in tasks that explicitly required the use of spatial information. However, there was a particular advantage for having a spatially organized mental map in a direction judgment task, especially for short distances where fine-grained knowledge was required. In contrast, there was no clear advantage for either group in a distance estimation task. These data are interpreted in the context of Huttenlocher's category adjustment model.
\end{abstract}

Studies have found that mental maps can be spatially (see, e.g., McNamara, 1986) or temporally (e.g., Clayton \& Habibi, 1991) organized. In the present article, we explore whether this affects the accuracy of spatial estimates per se. Specifically, we looked at performance on distance estimation and direction judgment tasks as a function of the need for fine- and coarse-grained information. This was done from the perspective of Huttenlocher, Hedges, and Duncan's (1991; Newcombe, Huttenlocher, Sandberg, Lie, \& Johnson, 1999) category adjustment theory of spatial memory.

When the organization of mental maps was first tested using primed recognition, the initial work suggested a spatial organization (e.g., McNamara, 1986; McNamara, Ratcliff, \& McKoon, 1984); however, when spatial and temporal proximity are deconfounded during learning, a temporal organization can be observed (e.g., Clayton \& Habibi, 1991), or at least a strong influence of temporal structure on retrieval (McNamara, Halpin, \& Hardy, 1992). The idea is that, under most conditions, there is a tendency to study spatially close items near one another in time. As a result, it is difficult to assess whether priming is due to spatial or temporal proximity. When these are separated, temporal information can play a prominent role in the structure of a mental map.

Curiel and Radvansky (1998) looked at priming when spatial and temporal proximity were deconfounded, varying map learning. For their naming group, a map location was indicated during learning, and the subject provided its name. For the pointing group, a name was provided dur-

\footnotetext{
We thank Jeremy Heckman for his assistance in data collection. This research was supported in part by an APSSC small grant awarded to the first author and a grant from the Army Research Institute, ARMYDASW01-99-K-0001, awarded to the second author. Correspondence concerning this article should be addressed to G. A. Radvansky, 107 Haggar, Department of Psychology, Notre Dame, IN 46556 (e-mail: radvansky.1@nd.edu).
}

ing learning, and the subject pointed to its map location. A temporal organization in primed recognition and free recall was found for the naming group, but a spatial organization was found for the pointing group. The interpretation was that people in the naming group had constructed a temporally organized mental map. Their learning task emphasized verbal information. In principle, the naming group could have performed perfectly without encoding any spatial information, learning only the order of the object names. Because of this lesser emphasis on precise spatial knowledge, the subjects' mental representations were more likely to involve temporal associations among the names. In comparison, subjects in the pointing group had constructed a spatially organized mental map. Their task emphasized spatial information, because each subject had to spatially guide a computer mouse to the correct location. Therefore, the subjects' mental representations were more likely to capture spatial details of the map.

Curiel and Radvansky (2002) had people use the learning procedure of the naming condition to memorize a map of a building. Temporal priming was observed. In a language comprehension task modeled after Rinck and Bower (1995), however, in which reading times to anaphoric sentences that referred to previously studied map locations were assessed, spatial effects were found. Also, reading times revealed that people were sensitive to spatial shifts from one map location to another. Finally, the subjects could organize their recall according to the map's spatial regions when they were instructed to do so. Thus, although the mental maps had a temporal organization, when the task required spatial information, the effects of spatial structure were observed. Still, the degree to which the use of spatial estimation is affected by the organization of the mental map remains unclear.

When people need to make spatial estimates, their accuracy is a function of the specific type of information that they have available. Because mental map organizations can vary, we decided to test whether these organi- 
zations would affect spatial estimation. In addition to differences in organization, there may also be differences in the amount of information available at different levels of detail. In Huttenlocher et al.'s (1991) categorical adjustment theory, people base their spatial estimates on two types of representations. One consists of highly detailed, fine-grained representations that capture metric spatial information. The other consists of less detailed, coarse-grained categorical representations that capture general characteristics, such as regions. The estimates people make from memory reflect the combined influences of these two representations.

The degree to which people use these representations varies in the extent to which the information in them is available. The more the information that is available at the fine-grained level, the more accurate a person will be, and vice versa. For spatially and temporally organized mental maps, the learning that produces a spatial organization should be more likely to leave a person with more detailed fine-grained representations than should the other type of learning. Thus, spatial tasks that demand more detailed information should be more sensitive to this difference than tasks that are less demanding. For these tasks, we used direction judgment and distance estimation, respectively, which are described in the next section.

\section{Experiment}

In this experiment, we explored the influence of mental map organization on performance accuracy, focusing on any decrements observed when a mental map is temporally organized and the task requires the detailed use of spatial information. We considered two possibilities. First, a temporal organization might reduce the level of fine-grained spatial information, and a task that requires it should reveal a performance decline. Second, the organization of the mental map might not have a strong influence on spatial judgments; spatial information, equally available in both situations, can be derived from the memory trace regardless of its organization.

The distance estimation task involved estimating the distance between two map locations. A memory psychophysics approach was used: Stevens' law (Stevens \& Galanter, 1957), in which physical magnitude estimates are described as a power function $\left(\Psi=k \Phi^{n}\right)$ that relates physical magnitude $(\Phi)$, to psychological experience $(\Psi)$, with a constant scaling factor $(k)$ and the slope of the function $(n)$ in $\log / \log$ coordinates. Distance estimation requires less detailed information, because a person can get by with general categorical information about two entities. For example, when driving in a city one may be aware that one is about 5 miles from one's hotel, but not be exactly sure of its direction. In this way, a person can primarily use coarse-grained information about distance, without needing fine-grained details about direction.

In the present experiment, we used maps explicitly divided into regions. Using these regions, as well as any subjective subdivisions, a person could use categorical information to make distance estimates. For example, two entities in remote areas could be reliably estimated as far away, whereas two entities in the same part of a region could be reliably estimated as near. Detailed knowledge of where the entities were within these categories was less critical.

The direction judgment task involved indicating the direction from one map entity to a second one. Accuracy was evaluated in absolute degrees of deviation between actual and estimated directions. Often, direction judgments of map locations result in errors of $10^{\circ}-30^{\circ}$ (Levine, Jankovic, \& Palij, 1982; Presson, DeLange, \& Hazelrigg, 1989; Presson \& Hazelrigg, 1984; Rossano, Warren, \& Kenan, 1995). This task requires more detailed information for accurate estimates. For example, if one knows that one is about 5 miles from a hotel, one will be able to successfully get back there only if one knows the direction in which to travel. Thus, finegrained information is needed.

Again, although the present experiment's map region information might be adequate for distance estimates, it is not as helpful for direction judgments. For entities that are far apart, the region information may suffice. Knowing the regions to which two entities belong as well as the relations between those regions can provide a person with general knowledge about the correct direction. Detailed finegrained information will not improve performance much in this situation. However, for entities close to one another in the same region, there is less likely to be categorical information that can aid a person in making estimates because both entities belong to the same spatial category. Under these circumstances, the availability of fine-grained spatial information will noticeably help performance.

\section{METHOD}

\section{Subjects}

Forty-eight students participated in the study and were compensated with either partial course credit or $\$ 5$. They were randomly assigned to one of three groups: a naming group $(n=16)$, a pointing group $(n=16)$, and a perception group $(n=16)$. Within each group, half were given the distance estimation task first and the rest were given the direction judgment task first. Data from 9 additional people were excluded because their performance on one or both tasks was highly erratic. This included 1 from the perception group, 4 from the pointing group, and 4 from the naming group.

\section{Materials}

A sample of a map used in the study is presented in Figure 1. It consisted of a $2 \times 2$ grid divided into quadrants that were approximately $6 \times 6 \mathrm{~cm}$. Each quadrant contained seven dots. The dots were placed so that 12 distances were represented twice, with the restriction that 6 unique distances were represented per region. These distances were: $0.3,1.4,2.0,2.6,3.7,4.8,5.4,6.0,7.1,7.7,8.2$, and $9.3 \mathrm{~cm}$. Within each group, each person studied a different map. However, the same set of 16 maps was used for all three groups.

The object names were three to seven letters long and had one or two syllables (e.g., brick and candle) with word frequencies ranging from 1 to $260(M=40)$ per million (Francis \& Kučera, 1982). For each person, 28 object names were randomly selected from the pool of 56 objects, and each was randomly assigned to a map location.

The temporal order of presentation was constant across learning for each person. This order was randomly determined for each per- 


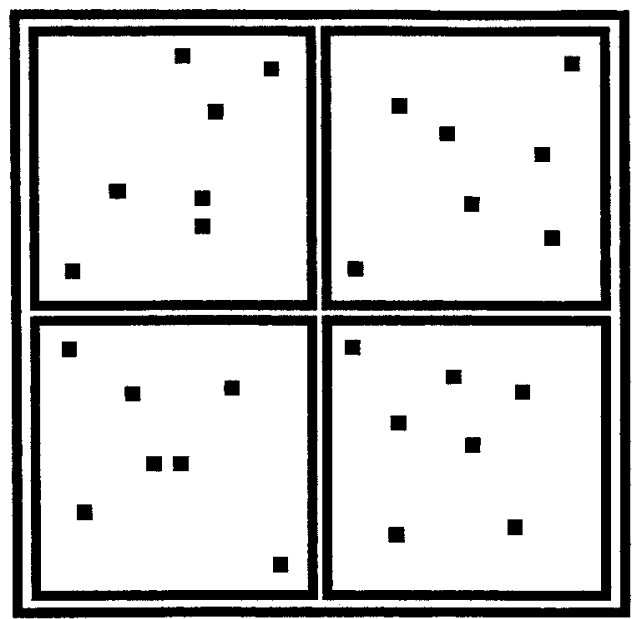

Figure 1. Map used in the experiment.

son with the restriction that objects from the same region were not presented consecutively. Thus, spatial and temporal proximity were deconfounded.

\section{Design and Procedure}

Learning. Students in the naming and pointing groups memorized a map using a study-test procedure. During study, map objects were presented one at a time. A dot blinked for $2 \mathrm{sec}$, and then the object name was presented next to it for $3 \mathrm{sec}$. The name was then erased, and the next dot began blinking. This continued until all of the object names were presented.

After all objects were presented, the subjects were given a cued recall test. The subjects in the naming group were cued with the location of an object by having a dot blink two times and turn red. Below the map, the question "What is the name of this object?" was presented. People typed their response into the computer. The pointing group was cued with the name of the object. Below the map the question "Where is the object?" was presented, in which the word object was replaced with an object name. The subjects used the mouse to move the cursor directly to a dot location and pressed the left mouse button to record the response location. For both groups, feedback consisted of displaying CORRECT or INCORRECT below the map along with the correct object name next to the correct location. After all of the questions were given, the subjects studied the map again. This study-test procedure was repeated until there were two errorless recalls.

After memorization, the direction judgment and distance estimation tasks were given. The perception group did not learn the map, but had it present on a second computer monitor.

Distance estimation. The distance estimation task comprised 96 trials of pairs of object names. Object pairs for each of the 12 distances were presented four times, twice in one order and twice in the reverse. On each trial, a pair of object names was presented halfway down the left side of the screen. A $0.3-\mathrm{cm}$-long horizontal line separated them. The subjects estimated the distance between the objects by adjusting the length of the line. Pressing the "M" key increased the length of the line, and pressing the " $Z$ " key decreased it. When satisfied with the estimate, the subject pressed the enter key. The trial order was randomly determined for each person.

Direction judgment. The direction judgment task consisted of 48 trials. Object pairs for each of the 12 distances were presented twice, once with each entity serving as the reference object, and once as the located object. On each trial, the reference object name was presented below a dot in the center of a circle that was $10 \mathrm{~cm}$ in diameter and was located in the center of the screen. The located object name was presented below the circle. People used the mouse to indicate the point on the circle that best represented the direction of the located object from the reference object. The trial order was randomly determined for each person.

\section{RESULTS}

For all analyses, a $p$ value of .05 is adopted, unless otherwise stated. Note that all distance estimates and direction judgments were made within map quadrants.

\section{Learning}

Subjects took an average of $5.5(S D=1.3)$ study-test cycles for memorization. The naming group took more $(M=5.9, S D=1.3)$ than did the pointing group $(M=$ $5.1, S D=1.1)\left[F(1,30)=4.19, M S_{\mathrm{e}}=1.46\right]$. Although this may indicate that location information is learned more quickly than identity information, these differences may also reflect how each type of information was tested, with typos possible (thereby leading to another study cycle) in the naming group.

\section{Distance Estimation}

The distance estimation data were first submitted to a $\log$ transformation. They were then trimmed by dropping trials greater than $2.5 \mathrm{SDs}$ from the mean. This eliminated $2 \%$ of the data. Each person's distance estimates were then submitted to a regression analysis with actual distance as the predictor. These analyses yielded exponent (slope), constant ( $y$-intercept), and $r^{2}$ values. The mean values for each group are presented in Table 1 .

The distance estimation data were analyzed in three separate 2 (order of presentation) $\times 2$ (group) mixed analyses of variance (ANOVAs) with slope, $y$-intercept, and $r^{2}$ as dependent variables. Because order was not significant in any of the analyses, it is not discussed further here. For the slopes, the main effect of group was significant $\left[F(2,42)=8.8, M S_{\mathrm{e}}=.06\right]$. The perceptual group's slope was greater than the slopes for the pointing $\left[F(1,30)=10.6, M S_{\mathrm{e}}=.08\right]$ and naming $[F(1,30)=15.5$, $\left.M S_{\mathrm{e}}=.11\right]$ groups, which did not differ $(F<1)$. For the $y$ intercepts, the perceptual group's was greater than the pointing group's, $\left[F(1,30)=16.32, M S_{\mathrm{e}}=.31\right]$, and marginally significantly greater than the naming group's

Table 1

Mean Distance Estimation and Direction Judgment (for Absolute Deviation Values) Data, With Standard Deviations

\begin{tabular}{|c|c|c|c|c|c|c|}
\hline & \multicolumn{6}{|c|}{ Group } \\
\hline & \multicolumn{2}{|c|}{ Perceptual } & \multicolumn{2}{|c|}{ Pointing } & \multicolumn{2}{|c|}{ Naming } \\
\hline & $M$ & $S D$ & $M$ & $S D$ & $M$ & $S D$ \\
\hline \multicolumn{7}{|l|}{ Distance Estimation } \\
\hline Exponent (slope) & .95 & .08 & .85 & .08 & .84 & .08 \\
\hline Constant ( $y$-intercept) & .11 & .17 & -.10 & .12 & -.04 & .20 \\
\hline$r^{2}$ & .94 & .06 & .87 & .06 & .87 & .06 \\
\hline Absolute error & 1.13 & 1.23 & 1.58 & .45 & 1.57 & .51 \\
\hline \multicolumn{7}{|l|}{ Direction Judgment } \\
\hline Absolute degrees of error & 11.9 & 6.4 & 13.3 & 3.1 & 16.5 & 5.6 \\
\hline
\end{tabular}


$\left[F(1,30)=3.2, M S_{\mathrm{e}}=.10, p=.08\right]$. The difference between the naming and pointing groups was not significant $\left[F(1,30)=2.3, M S_{\mathrm{e}}=.06, p>.05\right]$. For the $r^{2}$ values, the perceptual group was better than both the pointing $\left[F(1,30)=18.4, M S_{\mathrm{e}}=.04\right]$ and naming $[F(1,30)=$ $\left.20.2, M S_{\mathrm{e}}=.04\right]$ groups, which did not differ $(F<1)$. Thus, overall, the perception group outperformed the pointing and naming groups, which were equivalent.

Furthermore, distance estimation data were plotted against actual distance to see whether the groups differed in their estimates at particular distances. As can be seen in Figure 2A, there were no differences. These data indicate that a temporal mental map organization does not influence the accuracy of distance estimates. This is con- sistent with findings that some spatial information, such as Euclidean distance, may be stored in memory but is used only when needed (e.g., Rinck, Hähnel, Bower, \& Glowella, 1997).

Finally, the mean absolute deviations between the estimated and actual distances were calculated and are also presented in Table 1. The data were trimmed by eliminating trials that were $2.5 S D$ s beyond a person's mean. This eliminated $2 \%$ of the data. The distance estimation data were submitted to a 2 (Order of presentation) $\times 2$ (group) mixed ANOVA. None of the effects were significant (all $\left.F_{\mathrm{s}} \leq 1\right)$. Thus, there was no evidence that people in the memory groups were differentially able to estimate these magnitudes.

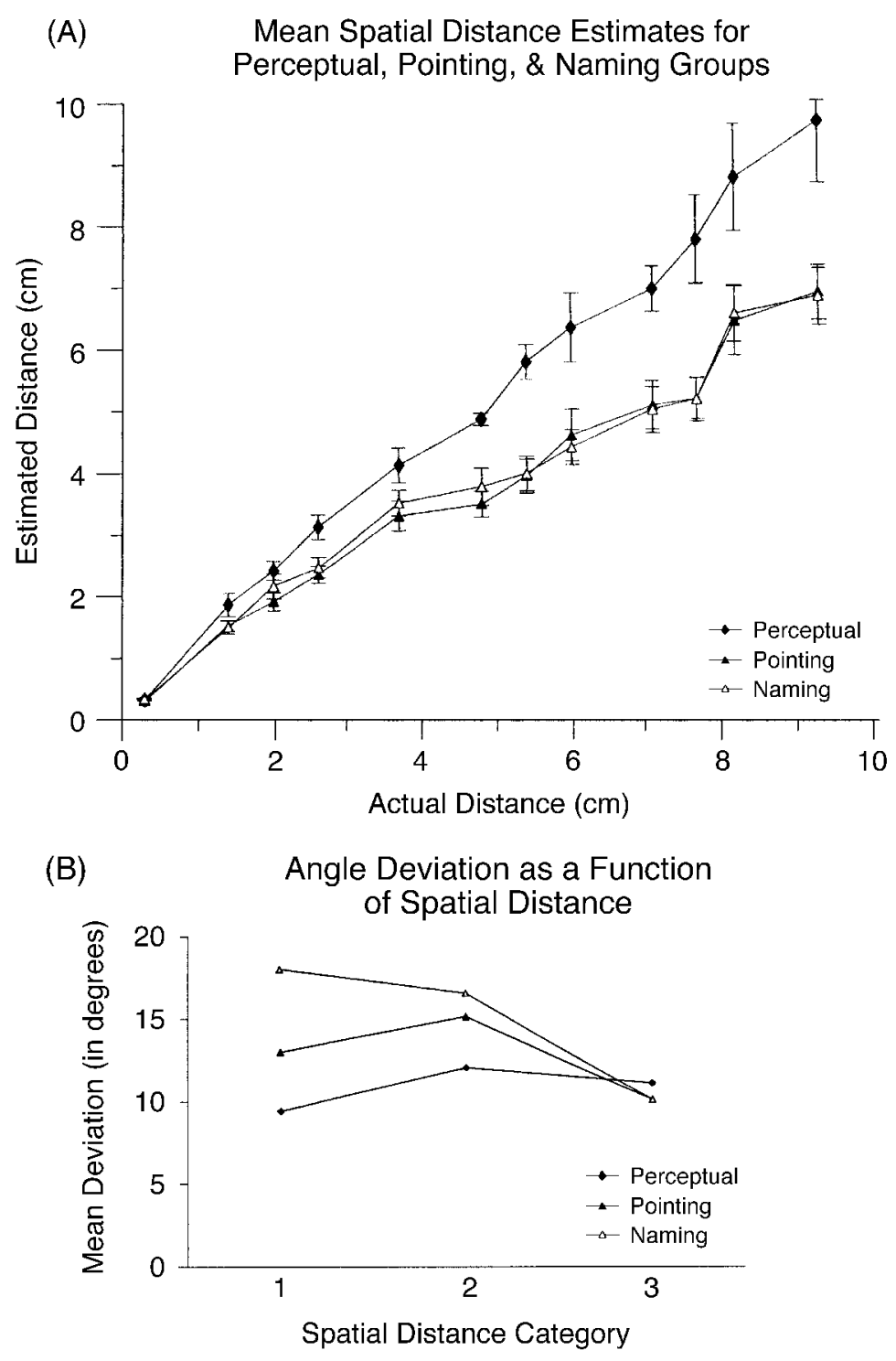

Figure 2. (A) Distance estimation and (B) direction judgment accuracy (for absolute deviation values) as a function of spatial distance. 


\section{Direction Judgment}

The direction judgment data were the mean absolute angular deviations between the estimated and actual directions and are presented in Table 1 . The data were trimmed by dropping trials that were $2.5 S D$ s beyond a person's mean. This eliminated $2 \%$ of the data. The direction judgment data were analyzed in a 2 (order of presentation) $\times 2$ (group) mixed ANOVA. Again, because all of the effects involving order were not significant, this factor is not considered further.

There was a significant main effect of group $[F(2,42)=$ $\left.3.23, M S_{\mathrm{e}}=89\right]$. The perceptual and pointing groups were more accurate than the naming group $[F(1,30)=4.65$, $M S_{\mathrm{e}}=168$, and $F(1,30)=4.08, M S_{\mathrm{e}}=83$, respectively] Moreover, the pointing and perceptual groups did not differ $(F<1)$. This pattern differs from that for the distance estimation data.

An additional analysis was done to see whether accuracy varied with distance. Because of the small number of observations per distance, we collapsed the data into three categories: the 4 shortest distances $(0.3,1.4$, 2.0 , and $2.6 \mathrm{~cm})$, the 4 intermediate distances $(3.7,4.8$, 5.4 , and $6.0 \mathrm{~cm})$, and the 4 greatest distances $(7.1,7.7$, 8.2 , and $9.3 \mathrm{~cm})$. The data were submitted to a 2 (group) $\times 3$ (distance) mixed ANOVA. There were significant main effects of group $\left[F(2,45)=3.13, M S_{\mathrm{e}}=\right.$ $248]$ and distance $\left[F(2,90)=10.35, M S_{\mathrm{e}}=274\right]$. Importantly, there was a significant interaction $[F(4,90)=$ 4.33, $\left.M S_{\mathrm{e}}=115\right]$, which can be seen in Figure 2B. The least deviation occurred at the greater distances and the greatest deviation occurred at the shorter distances with the naming group showing greater distortion than did the pointing $\left[F(1,30)=4.29, M S_{\mathrm{e}}=301\right]$ and perceptual $\left[F(1,30)=7.90, M S_{\mathrm{e}}=682\right]$ groups.

These results indicate that people have some knowledge of entity locations in their mental maps. However, the degree of detail depends on how the map was learned. Both learning groups stored general information about spatial location, which was consistent with the results of the distance estimation task. However, the pointing group stored more fine-grained information about the relations between spatially close objects. This is because this group needed to distinguish more precisely between entities during learning. Thus, the pointing group was more accurate when more fine-grained information was needed.

\section{DISCUSSION}

Previous research has found that the organization of a mental map reflects how the map was learned (Curiel \& Radvansky, 1998). When people learn a map by pointing to objects, a spatial organization is observed. In contrast, when people learn a map by naming objects, a temporal organization is observed. Furthermore, when the current task requires spatial information, that information can be readily used, even if it is inconsistent with the organization of the mental map (Curiel \& Radvansky, 2002).

The results of the present experiment show that memory can be more accurate when the mental map is spa- tially organized and is more likely to contain fine-grained information. This was observed in our direction judgment task and was most pronounced at short distances. As described in the introduction to the experiment, this is a portion of the map where fine-grained knowledge is most needed. For the distance estimation task, detailed information is not as important. Reasonable accuracy can be derived from coarse-grained categorical knowledge.

This different demand on spatial memory for categorical and fine-grained knowledge is captured by Huttenlocher's category adjustment model (Huttenlocher et al., 1991; Newcombe et al., 1999). According to this view, spatial information is stored in coarse- and fine-grained representations. Our maps were divided into four quadrants. However, it is possible that people subjectively divided the quadrants further into subregions, as can occur (e.g., McNamara, Hardy, \& Hirtle, 1989; Rinck et al., 1997). This categorical knowledge may be sufficient for accurate distance estimation. However, for direction judgment, general categorical information is not sufficient, especially for nearby entities. For entities in the same subjective category, more fine-grained spatial knowledge is needed. Such information was more available for the pointing group because of the need for more precise information to guide the mouse cursor during learning. In contrast, for the naming group, although spatial information was helpful, the task could potentially have been performed with no reference to spatial information at all, only to the temporal sequence. Thus, spatial detail information is likely to be encoded less well in this condition. In other words, the different training criteria in the two groups not only led to differences in mental organization of information (a difference in quality), but also to differences in the amount of finegrained information encoded into the mental map (a difference in quantity).

In conclusion, the degree of detail encoded into a mental map can influence the latter use of spatial information. This was observed in cases where more finegrained spatial knowledge was needed, as in our direction judgment task. If this information is less critical, then more general, categorical knowledge may be adequate. This was truer of our distance estimation task. Thus, it is possible for the mental map to contain some spatial information without necessarily being spatially organized. Consistent with the categorical adjustment theory (Huttenlocher et al., 1991; Newcombe et al., 1999), people were more likely to be able to use finegrained information when it was adequately encoded. More generally, this line of research is consistent with the idea that information is stored at different levels of detail, and that the influences of these multiple representations combine to produce the resulting pattern of performance.

\section{REFERENCES}

Clayton, K., \& HABiBi, A. (1991). Contribution of temporal contiguity to the spatial priming effect. Journal of Experimental Psychology: Learning, Memory, \& Cognition, 17, 263-271. 
Curiel, J. M., \& RAdVAnsky, G. A. (1998). Mental organization of maps. Journal of Experimental Psychology: Learning, Memory, \& Cognition, 24, 202-214.

Curiel, J. M., \& Radvansky, G. A. (2002). Mental maps in memory retrieval and comprehension. Memory, 10, 113-126.

FRANCIS, W. N., \& KUĈERA, H. (1982). Frequency analysis of English usage. Boston: Houghton Mifflin.

Huttenlocher, J., Hedges, L. V., \& Duncan, S. (1991). Categories and particulars: Prototype effects in estimating spatial location. Psychological Review, 98, 352-376.

LeVine, M., Jankovic, I. N., \& PAliJ, M. (1982). Principles of spatial problem solving. Journal of Experimental Psychology: General, 111, $157-175$

MCNAmARA, T. P. (1986). Mental representations of spatial relations. Cognitive Psychology, 18, 87-121.

MCNamara, T. P., Halpin, J. A., \& Hardy, J. K. (1992). Spatial and temporal contributions to the structure of spatial memory. Journal of Experimental Psychology: Learning, Memory, \& Cognition, 18, 555564.

McNamara, T. P., Hardy, J. K., \& Hirtle, S. C. (1989). Subjective hierarchies in spatial memory. Journal of Experimental Psychology: Learning, Memory, \& Cognition, 15, 211-227.

McNamara, T. P., RatclifF, R., \& McKoon, G. (1984). The mental representation of knowledge acquired from maps. Journal of Experimental Psychology: Learning, Memory, \& Cognition, 10, 723-732.

Newcombe, N., Huttenlocher, J., SAndberg, E., Lie, E., \& John-
SON, S. (1999). What do misestimations and asymmetries in spatial judgment indicate about spatial representation? Journal of Experimental Psychology: Learning, Memory, \& Cognition, 25, 986-996.

Presson, C. C., Delange, N., \& HazelrigG, M. D. (1989). Orientation specificity in spatial memory: What makes a path different from a map of a path? Journal of Experimental Psychology: Learning, Memory, \& Cognition, 15, 887-897.

Presson, C. C., \& HAZELRIGG, M. D. (1984). Building spatial representations through primary and secondary learning. Journal of Experimental Psychology: Learning, Memory, \& Cognition, 10, 716722.

RINCK, M., \& Bower, G. H. (1995). Anaphora resolution and the focus of attention in situation models. Journal of Memory \& Language, 34, 110-131.

Rinck, M., Hähnel, A., Bower, G. H., \& Glowella, U. (1997). The metrics of spatial situation models. Journal of Experimental Psychology: Learning, Memory, \& Cognition, 23, 622-637.

Rossano, M. J., Warren, D. H., \& Kenan, A. (1995). Orientation specificity: How general is it? American Journal of Psychology, 108, 359-380.

SteVens, S. S., \& Galanter, E. H. (1957). Ratio scales and category scales for a dozen perceptual continua. Journal of Experimental Psychology, 54, 377-411.

(Manuscript received June 29, 2001; revision accepted for publication February 26, 2003.) 